\title{
Helmet Exhalation Capture System (HECS) Sizing Evaluation for an Advanced Space Suit Portable Life Support System
}

\author{
Heather L. Paul (NASA - Johnson Space Center) \\ Glenn M. Waguespack (Geocontrol Systems, Inc.) \\ Thomas H. Paul (Jacobs Technology) \\ Bruce C. Conger (Hamilton Sundstrand)
}

\subsection{Abstract}

As part of NASA's initiative to develop an advanced portable life support system (PLSS), a baseline schematic has been chosen that includes gaseous oxygen in a closed circuit ventilation configuration. Supply oxygen enters the suit at the back of the helmet and return gases pass over the astronaut's body to be extracted at the astronaut's wrists and ankles through the liquid cooling and ventilation garment (LCVG). The extracted gases are then treated using a rapid cycling amine (RCA) system for carbon dioxide and water removal and activated carbon for trace gas removal before being mixed with makeup oxygen and reintroduced into the helmet. Thermal control is provided by a suit water membrane evaporator (SWME).

As an extension of the original schematic development, NASA evaluated several Helmet Exhalation Capture System (HECS) configurations as alternatives to the baseline. The HECS configurations incorporate the use of full contact masks or non-contact masks to reduce flow requirements within the PLSS ventilation subsystem. The primary scope of this study was to compare the alternatives based on mass and volume considerations; however other design issues were also briefly investigated. This paper summarizes the results of this sizing analysis task.

\subsection{Evaluated Configurations}

Four unique PLSS configurations were assessed in this study: modified baseline, open loop full-contact mask, closed loop full-contact mask, and closed loop noncontact mask. These PLSS conceptual designs are described in the following discussion.

Differences between the four evaluated PLSS configurations only occur within the ventilation systems and primary oxygen systems. The PLSS designs share identical non-ventilation technologies. These common technologies include the thermal control system, power supply system, and emergency oxygen supply system.

Excess heat is removed from the suit interior via a series of water tubes within the Liquid Cooling and Ventilation Garment (LCVG). The flowing water recirculates through a Suit Water Membrane Evaporator (SWME) and cools the flow through the evaporation of expendable water through a porous membrane. The cooling water flow is driven by a $33.25 \mathrm{~W}$ pump.

Electric resistance heaters provide any necessary heating.

Electric power is provided by a lithium-ion battery pack.

\subsection{Modified Baseline Configuration}

The original baseline configuration for this study, as shown in Figure 1, was developed through the Constellation Suit Element Portable Life Support System (PLSS) Schematic Selection Study [1]. In this configuration, oxygen is introduced into the rear of the helmet. A portion of this $\mathrm{O}_{2}$ is inhaled by the astronaut. The exhaled gases are entrained in the remainder of the $\mathrm{O}_{2}$ flow, which proceeds out of the helmet and through the suit interior. These gases exit the suit interior at the astronaut's extremities through the LCVG and are then directed through a rapid cycling amine (RCA) unit, which removes excess $\mathrm{CO}_{2}$ and $\mathrm{H}_{2} \mathrm{O}$, and an activated carbon filter, which removes trace contaminants. The treated $\mathrm{O}_{2}$ stream then mixes with makeup $\mathrm{O}_{2}$ and reenters the helmet. In the original baseline configuration, which uses an Apollo/Shuttle-based helmet design, $\mathrm{O}_{2}$ flow rate of $170 \mathrm{alpm}(6 \mathrm{acfm})$ is required to ensure adequate $\mathrm{CO}_{2}$ removal [2] so that the inhalation of excessive $\mathrm{CO}_{2}$ concentrations is avoided. This is known as the $\mathrm{CO}_{2}$ wash-out requirement. A fan is used to overcome the ventilation system pressure losses corresponding to the 170 alpm (6 acfm) flow. 


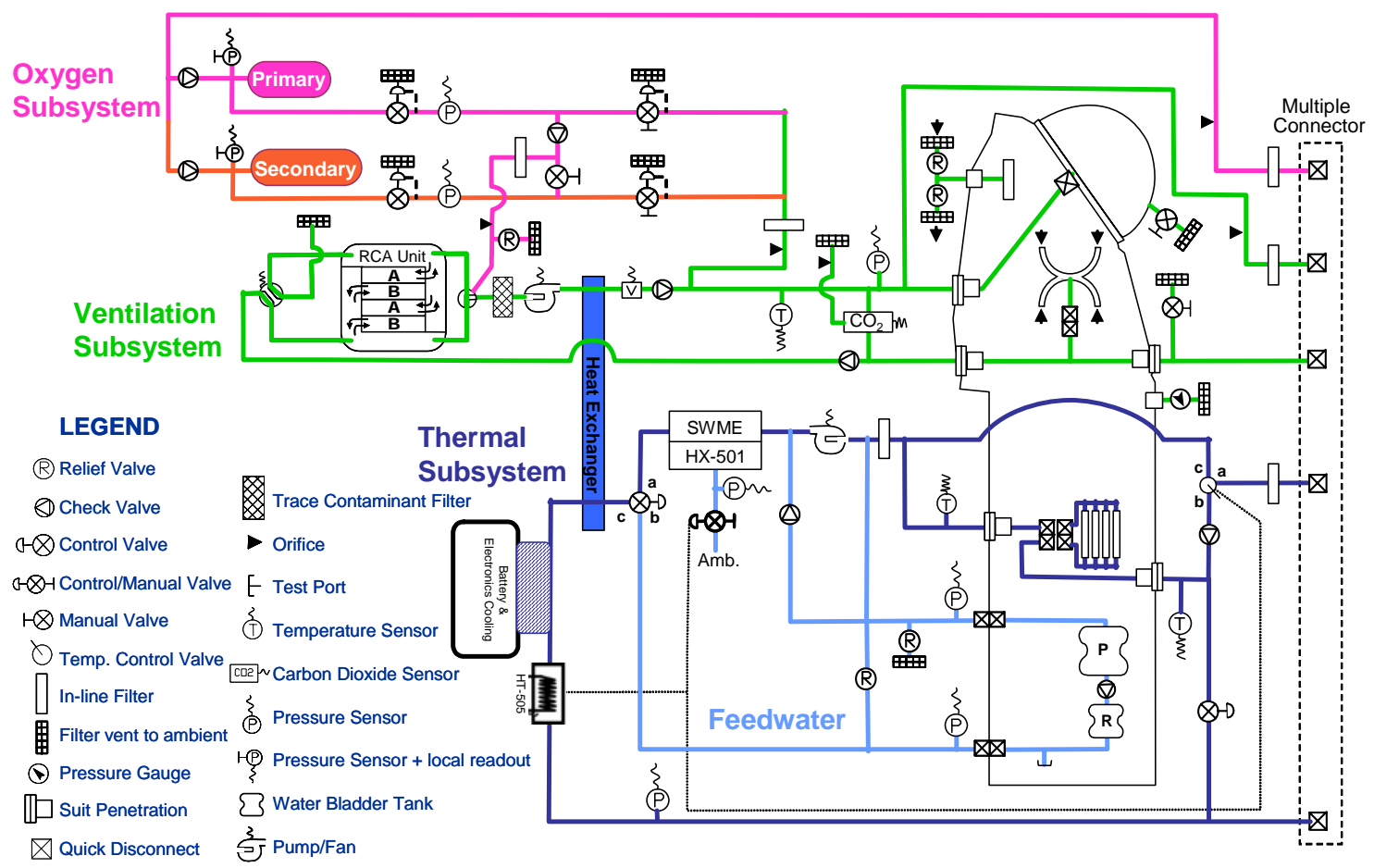

Figure 1: Schematic diagram of baseline PLSS (original and modified).

For purposes of this study, the original baseline configuration was modified by replacing the helmet with a newer hemispherical helmet design, which only requires $113 \mathrm{alpm}$ (4 acfm) oxygen inlet flow for $\mathrm{CO}_{2}$ wash-out, by replacing the original fan with an air bearing fan (ABF) [3], and by resizing the RCA unit to accommodate the differing ventilation flow rate and $\mathrm{CO}_{2}$ concentration. Since the differences between the original and modified baseline configurations consist only of differing helmet and ventilation fan designs, the schematic depicted in Figure 1 represents both the original and modified baseline configurations.

\subsection{Open Loop Full Contact Mask}

The open loop configuration has all exhaled gases dumped overboard, thus eliminating the need for $\mathrm{CO}_{2}$, $\mathrm{H}_{2} \mathrm{O}$, and trace gas treatment components. This configuration is shown in Figure 2. The need for a ventilation fan is also eliminated due to the exclusion of ventilation gas treatment components. Because a significant amount of $\mathrm{O}_{2}$ is present in the exhalation gases, open loop systems waste the $\mathrm{O}_{2}$ that would otherwise be recirculated in a closed loop system. The mass and volume associated with the storage of this extra $\mathrm{O}_{2}$ offsets the mass and volume reductions produced by eliminating the fan, RCA, and activated carbon canister. Because breathing masks prevent mixing of exhalation gases with inhalation gases, the extra flow requirement needed for $\mathrm{CO}_{2}$ washout in non- mask designs is not required, providing lower $\mathrm{O}_{2}$ consumption than an equivalent open loop non-mask design.

\subsection{Closed Loop Full Contact Mask}

The closed-loop, full-contact mask configuration shown in Figure 3 reduces the total $\mathrm{O}_{2}$ storage requirement by combining the lower $\mathrm{O}_{2}$ flow requirement of a breathing mask with the $\mathrm{O}_{2}$ consumption efficiency of a recirculating system. This system contains the same components as the modified baseline configuration; however, the addition of a breathing mask reduces the required flowrate from the $\mathrm{CO}_{2}$ wash-out requirement of the modified baseline configuration to the average respiratory ventilation rate at a design metabolic rate of $600 \mathrm{~W}$, which was selected to ensure that the sized components can accommodate transient metabolic rate peaks. This reduction in $\mathrm{O}_{2}$ flowrate reduces the mass and volume associated with the $\mathrm{O}_{2}$ storage and RCA systems, as well as the ventilation fan mass and volume. The lower fan power permits use of a smaller battery and directly reduces the cooling load attributed to electric power consumption within the suit environment. An indirect cooling load reduction results from the decrease in battery heating associated with the reduction of electric load. 


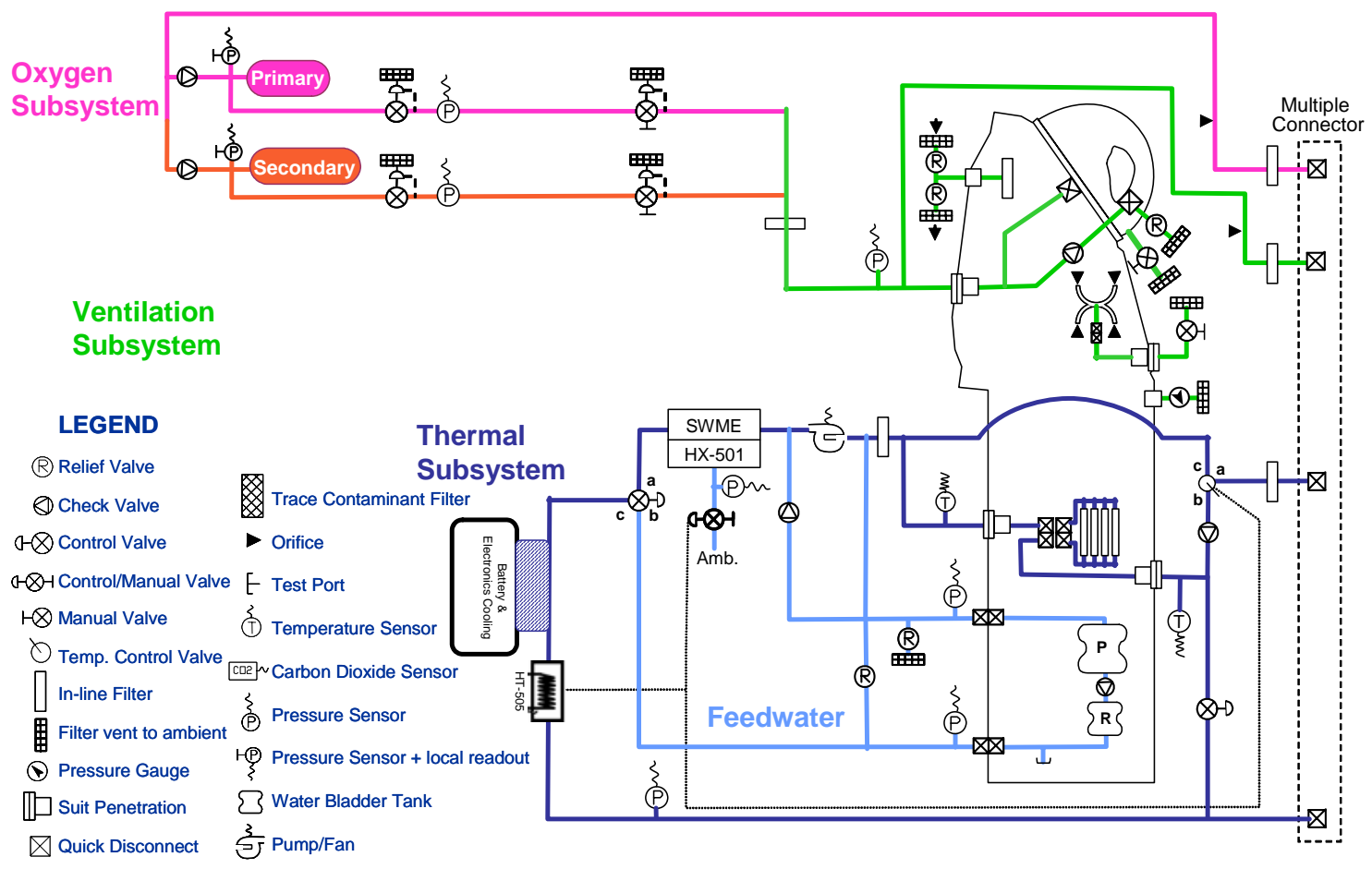

Figure 2: Schematic diagram of open loop full contact mask configuration.

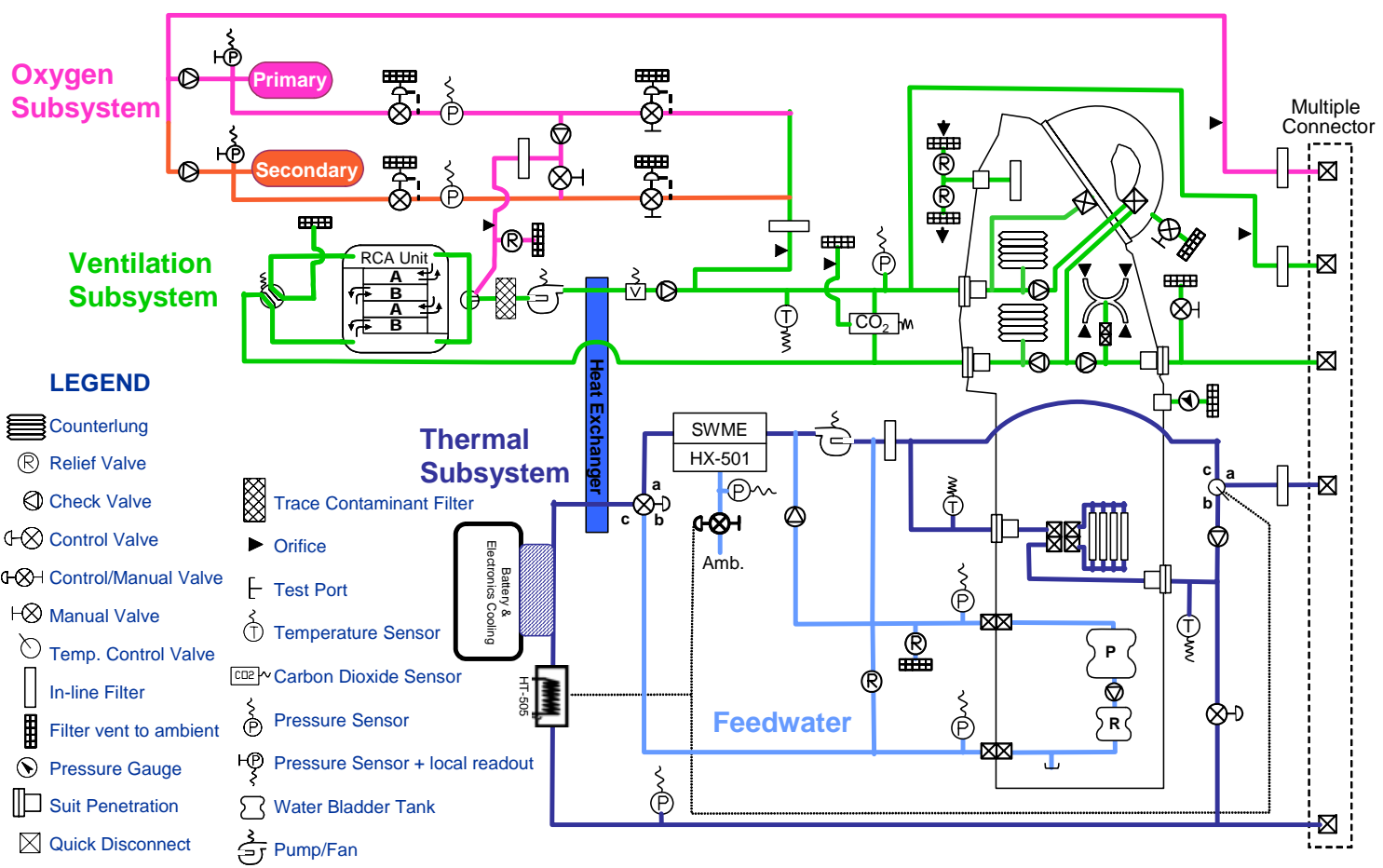

Figure 3: $\quad$ Schematic diagram of the closed loop full contact mask configuration with decoupled constant fan speed counterlungs.

Because breathing is a cyclic process consisting of alternating inhalation and exhalation, the ventilation system must provide transient storage areas, or reservoirs, for exhaled and inhaled gases. For nonmask systems, the spacesuit acts as a common reservoir. For open-loop mask systems, the $\mathrm{O}_{2}$ supply tank is the inhalation reservoir and the space environment is the exhalation reservoir. For a closedloop mask system, however, the constrained flow path necessitates the inclusion of at least one expandable counterlung (accumulator). Three different counterlung configurations were considered for this study, all of which use two separate counterlungs: decoupled constant fan speed counterlungs, coupled fanless 
counterlungs, and breathing bags. A sizing analysis was performed only on the decoupled constant fan speed counterlung configuration.

\subsubsection{Decoupled Constant Fan Speed Counterlungs}

For the decoupled constant fan speed counterlung configuration two bellows-type counterlungs are used: one to store exhaled gases and the other to store treated inhalation gas. The RCA unit, ventilation fan, activated carbon canister, and makeup $\mathrm{O}_{2}$ inlet are located between the counterlungs. Each counterlung is mechanically independent of the other so that the ventilation fan can move gases from the exhalation counterlung to the inhalation counterlung at a constant speed.

As the astronaut exhales, the exhalation counterlung fills. Concurrently, the ventilation fan continuously transfers the exhaled gases through the RCA and activated carbon canister into the inhalation counterlung. Makeup $\mathrm{O}_{2}$ is added to this mixture as needed. The astronaut then inhales the accumulated $\mathrm{O}_{2}$ from the inhalation counterlung, completing the cycle.

\subsubsection{Coupled Fanless Counterlungs}

Two bellows-type counterlungs are mechanically coupled such that they both expand and contract simultaneously. The RCA unit, activated carbon canister, and makeup $\mathrm{O}_{2}$ inlet are located between the counterlungs. No ventilation fan is used in this configuration. As the astronaut exhales, both counterlungs are simultaneously filled. The gases filling the inhalation counterlung first pass through the exhalation counterlung, RCA, and activated carbon canister. When the astronaut inhales, both counterlungs simultaneously compress. The suction of gases from the inhalation counterlung causes it to compress. This compressive force is exerted on the exhalation counterlung, due to the mechanical coupling, pushing the exhaled gases remaining in the exhalation counterlung through the rest of the system. Because no fan is used to overcome the system pressure drop, the force required from the astronaut's lungs is greater than in the fan-driven configurations.

\subsubsection{Breathing Bags}

The breathing bag concept is very similar to the "Decoupled Constant Fan Speed Counterlungs" configuration, except that the bellows-type counterlungs are replaced with flat breathing bags. The breathing bags would be mounted inside the suit, distributed as a liner.

\subsection{Closed Loop Non-Contact Mask}

If a funnel-like exhaust mask or non-contact mask is mounted in front of the astronaut's mouth and nose, the gases exiting the suit environment will have a higher $\mathrm{CO}_{2}$ concentration. By removing the $\mathrm{CO}_{2}$ from the helmet environment in its most concentrated state, the flowrate needed for $\mathrm{CO}_{2}$ wash-out reduces to $85 \mathrm{alpm}$ ( 3 acfm) for a hemispherical helmet design, as shown by the computational fluid dynamics (CFD) model presented in Section 3.3.1.4. This drop in $\mathrm{O}_{2}$ flowrate reduces the mass and volume associated with the $\mathrm{O}_{2}$ storage and RCA systems, as well as the ventilation fan mass and volume. The lower fan power permits use of a smaller battery and directly reduces the cooling load attributed to electric power consumption within the suit environment. An indirect cooling load reduction results from the decrease in battery heating associated with the reduction of electric load. A schematic representing this system is shown in Figure 4. Since the inhaled and exhaled gases can freely spill over into the full suit interior, the suit acts as a common reservoir, similar to configurations in which no mask is used. This eliminates the need for counterlungs, along with their associated masses and volumes.

\subsection{Analysis and Results}

The mass and volume results for the modified baseline configuration, open and closed loop full contact mask configurations, and non-contact mask configuration are shown in Tables 1 and 2. Only the subsystems that change between configurations are reported. The masses and volumes of the varying subsystems that are considered part of the portable life support system (PLSS) are summed to form the PLSS subtotals. (Note that a breathing mask is not characterized as a PLSS component.) Similarly, the masses and volumes of all varying subsystems are summed to form the suit system subtotals. Mass and volume packaging factors of 1.3 and 2.3, respectively, are multiplied to the subtotals to form packaging-adjusted subtotals. The PLSS and suit system totals consist of the packaging-adjusted masses and volumes of all components of the PLSS and of the total suit system, including the subsystems not explicitly reported in Tables 1 and 2 . 


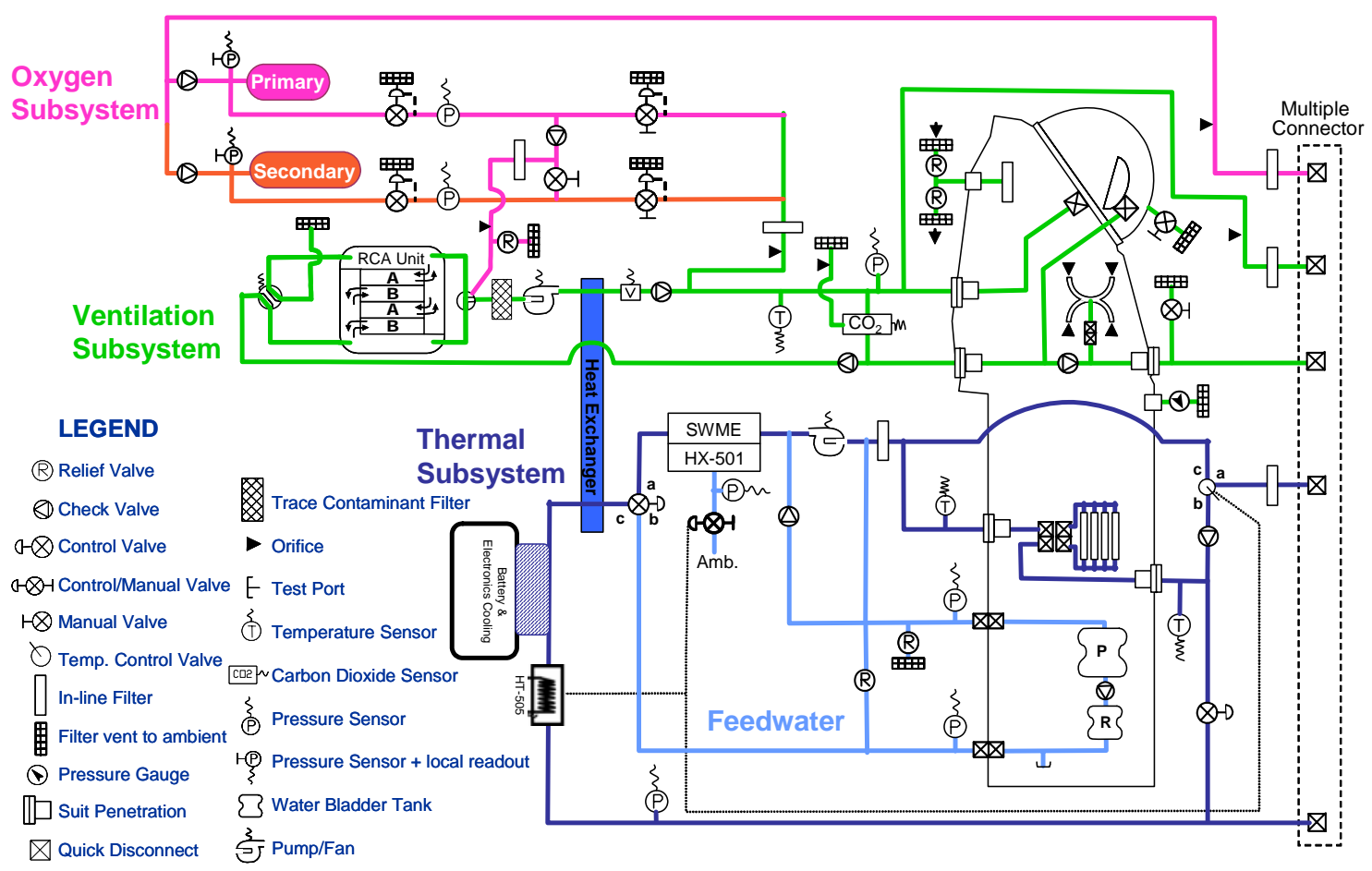

Figure 4: Schematic of a closed loop non-contact mask PLSS configuration.

Table 1: Sized Masses for Modified Maseline and Mask-Based PLSS Configurations

\section{Total Mass (kg)}

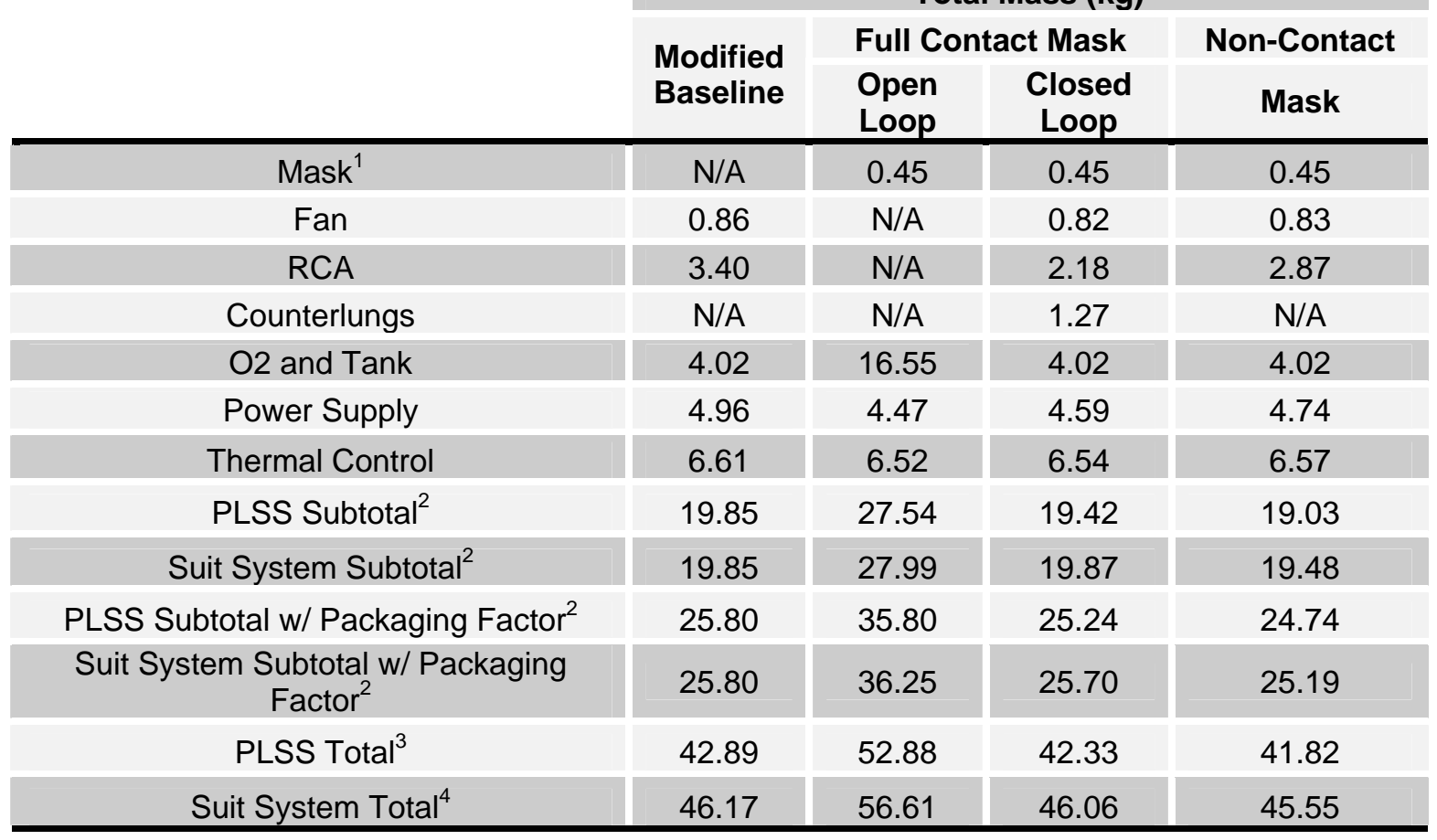

${ }^{1}$ Mask is not included in PLSS (sub)totals

${ }^{3}$ Includes all PLSS components

${ }^{2}$ Includes listed components only

${ }^{4}$ Includes all suit system components 
Table 2: Sized Volumes for Modified Baseline and Mask-Based PLSS Configurations

\begin{tabular}{|c|c|c|c|c|}
\hline & \multicolumn{4}{|c|}{ Total Volume $\left(\mathrm{m}^{3}\right)$} \\
\hline & \multirow{2}{*}{$\begin{array}{l}\text { Modified } \\
\text { Baseline }\end{array}$} & \multicolumn{2}{|c|}{ Full Contact Mask } & \multirow{2}{*}{$\begin{array}{l}\text { Non-Contact } \\
\text { Mask }\end{array}$} \\
\hline & & Open Loop & Closed Loop & \\
\hline Mask $^{1}$ & N/A & 0.00041 & 0.00041 & 0.00041 \\
\hline Fan & 0.00030 & N/A & 0.00028 & 0.00029 \\
\hline RCA & 0.0029 & N/A & 0.0013 & 0.0022 \\
\hline Counterlungs & N/A & N/A & 0.0052 & N/A \\
\hline O2 and Tank & 0.0033 & 0.0198 & 0.0033 & 0.0033 \\
\hline Power Supply & 0.0037 & 0.0034 & 0.0034 & 0.0036 \\
\hline Thermal Control & 0.0043 & 0.0043 & 0.0043 & 0.0043 \\
\hline PLSS Subtotal $^{2}$ & 0.0146 & 0.0274 & 0.0178 & 0.0137 \\
\hline Suit System Subtotal ${ }^{2}$ & 0.0146 & 0.0278 & 0.0182 & 0.0141 \\
\hline PLSS Subtotal w/ Packaging Factor ${ }^{2}$ & 0.0337 & 0.0629 & 0.0410 & 0.0315 \\
\hline $\begin{array}{l}\text { Suit System Subtotal w/ Packaging } \\
\text { Factor }^{2}\end{array}$ & 0.0337 & 0.0633 & 0.0414 & 0.0319 \\
\hline PLSS Total $^{3}$ & 0.0585 & 0.0877 & 0.0658 & 0.0563 \\
\hline Suit System Total $^{4}$ & 0.0935 & 0.1231 & 0.1012 & 0.0916 \\
\hline
\end{tabular}

The non-contact mask configuration provides minor mass and volume savings compared to the alternatives, mainly due to the fact that the sizes of the ventilation fan and RCA unit could be reduced from the modified baseline without wasting $\mathrm{O}_{2}$ consumables as exhaust or requiring the added mass and volume of counterlungs.

Compared to the modified baseline configuration, the closed loop full contact mask configuration uses slightly less mass but more volume. The lower mass results from the smaller ventilation fan and RCA units, due to lower $\mathrm{O}_{2}$ flow requirement. The volume increases, however, because of the addition of counterlungs.

The open loop full-contact mask option requires the most mass and volume because the extra mass and volume associated with storing the required excess $\mathrm{O}_{2}$ overcomes the mass and volume savings associated with omitting the breathing gas recirculation and treatment components.

Based on these results, none of the mask-based configurations offer significant mass and volume benefits over the modified baseline configuration.

The specific analyses of the primary subsystems that vary according to configuration are documented in the following discussion.

\section{$3.1 \mathrm{O}_{2}$ Supply Sizing}

For all closed-loop configurations the $\mathrm{O}_{2}$ supply system is sized to replenish the oxygen at its metabolic consumption rate [4], plus any system leakages and ullages. The $\mathrm{O}_{2}$ supply for the open-loop mask configuration is sized to accommodate the average lung ventilation rate [5].

\subsection{Counterlung Sizing}

For sizing the counterlungs for a closed loop full contact mask configuration, data was obtained from rebreather manufacturer Halcyon [6]. The masses and volumes of the Halcyon RB80 counterlung components were adapted to size a PLSS counterlung conceptual design that utilizes two decoupled bellows in conjunction with a constant-speed ventilation fan. 


\subsection{Fan Sizing}

Determining ventilation fan sizes for the non-baseline configurations consists of the following procedure:

1. Determine the required ventilation flow rate.

2. Determine the system pressure drop associated with the ventilation flow rate found in step 1 above.

3. Scale a known fan, in accordance with similarity laws, to the new pressure and flow requirements in order to estimate the fan's mass and volume.

Each ventilation fan is sized to support $600 \mathrm{~W}$ (2048 Btu/h) metabolic rate activity at a suit pressure of $30 \mathrm{kPa}$ (4.3 psia).

The mass, volume, and power requirements for the ventilation fan in the closed-loop, mask-based PLSS designs were calculated using the similarity-based "fan laws" [10] to scale the Air Bearing Fan (ABF) [3] to proposed PLSS requirements. The fan volume is scaled as a constant-length cylinder, whose diameter is proportional to the fan impeller size. The fan mass is assumed linearly proportional to its volume. The fan power is calculated from the corresponding flow rate and pressure lift, assuming a fan efficiency of 0.15 , which is the calculated efficiency of the ABF when applied to Shuttle EMU conditions [3].

The ventilation system pressure drops for all configurations are calculated using the pressure/flow characteristics of the current Shuttle EMU [8] as a reference. The ventilation system pressure drops are calculated under the assumption that the system pressure drop is proportional to the gas flow rate raised to the power of 1.75 , which derives from standard flow analysis relationships when accounting for frictional effects using the Blasius correlation [9].

\subsubsection{Flow Rate Determination}

The ventilation flow rate required for each configuration depends either on the $\mathrm{CO}_{2}$ wash-out capabilities of the system or the lung ventilation rate of the astronaut, depending on whether or not untreated exhalation gases are allowed to mix with the inhalation gases. Table xxx shows the flow rates required for each of the configurations evaluated.
Table xxx: Flowrate levels estimated for the configurations evaluated:

\begin{tabular}{|c|c|c|c|c|}
\hline & $\begin{array}{c}\text { Baseline } \\
\text { Hemispherical } \\
\text { Helmet }\end{array}$ & $\begin{array}{c}\text { Open } \\
\text { Loop } \\
\text { Full } \\
\text { Contact } \\
\text { Mask }\end{array}$ & $\begin{array}{c}\text { Closed } \\
\text { Loop } \\
\text { Full } \\
\text { Mask }\end{array}$ & $\begin{array}{c}\text { Closed } \\
\text { Loop } \\
\text { Non- } \\
\text { Contact } \\
\text { Mask }\end{array}$ \\
\hline $\begin{array}{c}\text { Flowrate - } \\
\text { actual cubic } \\
\text { feet per } \\
\text { minute (acfm) }\end{array}$ & 4 & $1.7^{*}$ & $1.7^{*}$ & $3^{* \star}$ \\
\hline
\end{tabular}

* Metabolic rate dependent - 1.7 represents flow requirement at $600 \mathrm{~W}(\sim 2000 \mathrm{Btu} / \mathrm{hr})$ metabolic rate (Bioastronautics Data Book)

** Computational Fluid Dynamics (CFD) evaluation determined non-contact mask flow requirement

\subsubsection{CFD Evaluation for the Closed Loop Non- Contact Mask}

Though providing a method to remove higher- $\mathrm{CO}_{2}-$ concentration gases from the helmet, the closed loop non-contact mask does not prevent the exhaled gases from mixing with the supply $\mathrm{O}_{2}$ entering the helmet. Therefore, $\mathrm{CO}_{2}$ washout is a requirement for this configuration. In order to assess the $\mathrm{O}_{2}$ flowrate needed to adequately satisfy the $\mathrm{CO}_{2}$ washout requirement, a computational fluid dynamics (CFD) study was performed.

For the CFD analysis, the first task was to generate the required geometry. A recent design of the Mark III suit geometry was obtained as a STEP (STandard for the Exchange of Product model data) file from ESCG designers. This suit geometry was created from a laser scan of an actual prototype suit. Suit arms and a canopy were also provided in the same file. A picture of the front of the geometry is shown in Figure 5. The model extended to approximately the wrist of the human, and down to the waist or the lower extent of the hard upper torso (HUT). Flow inputs for providing the supply breathing gas were placed along the back upper curve of the HUT as shown in Figure 6. This area was subdivided into numerous surfaces to allow for flexibility in deciding where the incoming flow could be. These can be seen in Figure 6.

The human geometry was created from a couple of different sources. The body below the head was taken from current crew quarters CFD simulations using a $95^{\text {th }}$ percentile male. The head of the human was taken from 
the same STEP file along with the HUT and arms. These two pieces were connected together and are shown in Figure 7.

To simulate a human breathing in and out inside the EMU, surfaces for the mouth and nose were created. These can be seen in Figure 8.

The complete geometry is shown in Figure 9 with a transparent canopy and arms.

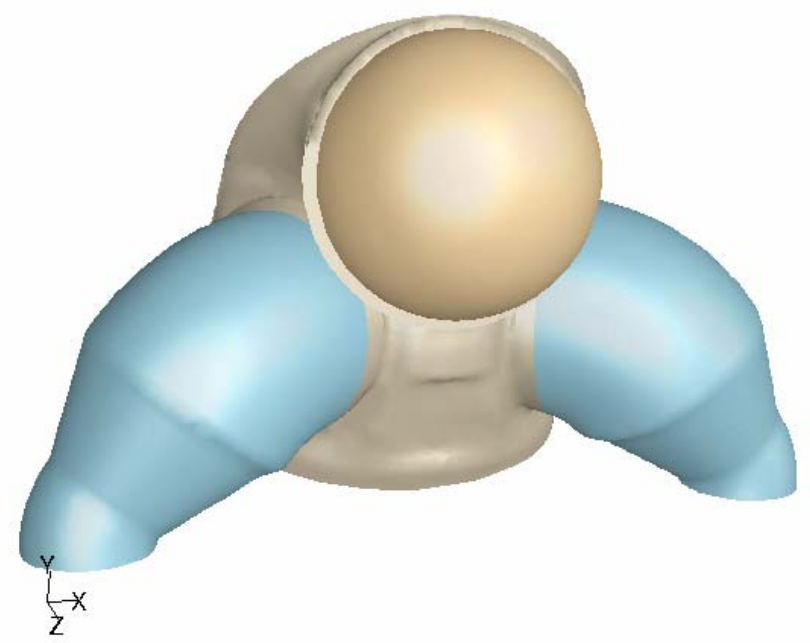

Figure 5: Front view of EMU geometry

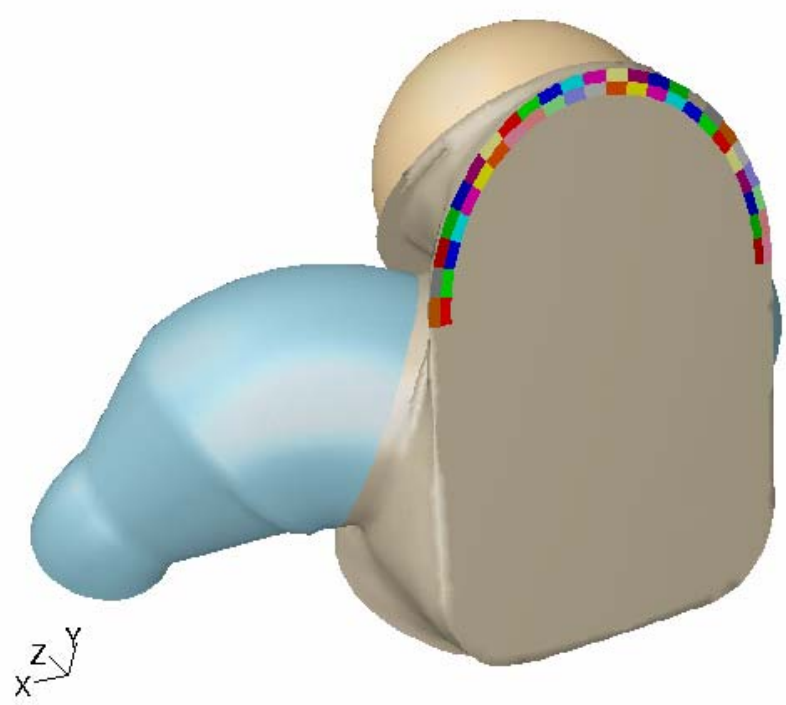

Figure 6: Rear view of HUT with inlets along the top

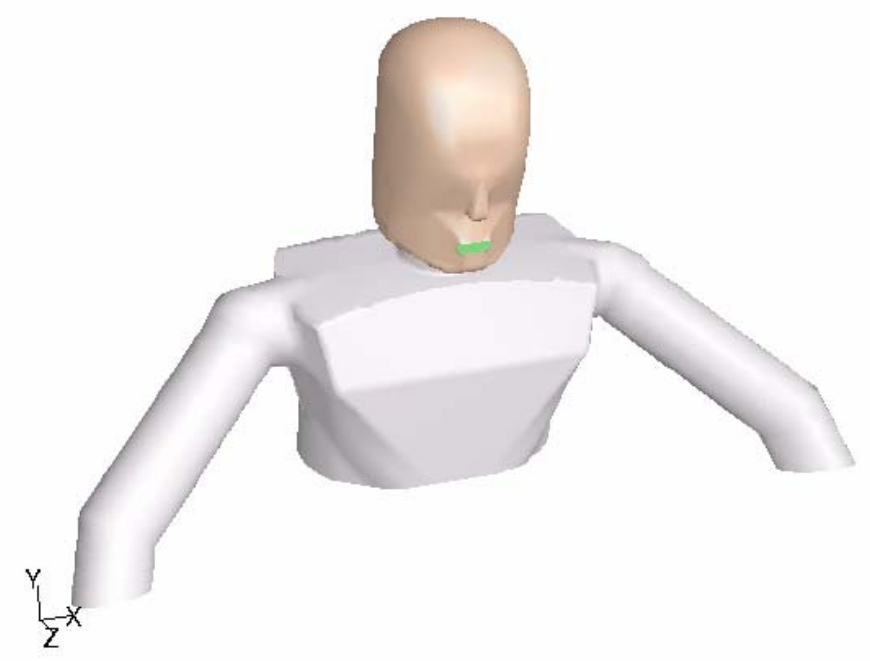

Figure 7: Human surface geometry

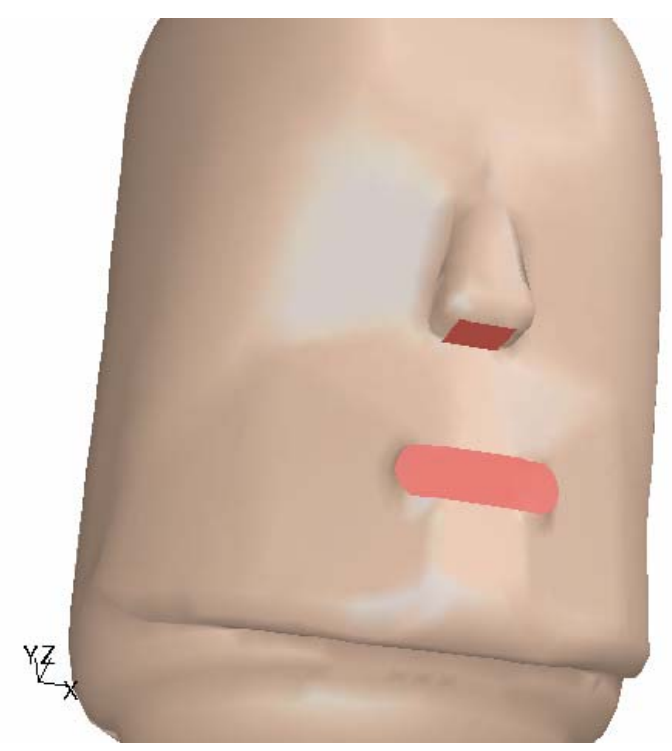

Figure 8: Human mouth and nose surfaces

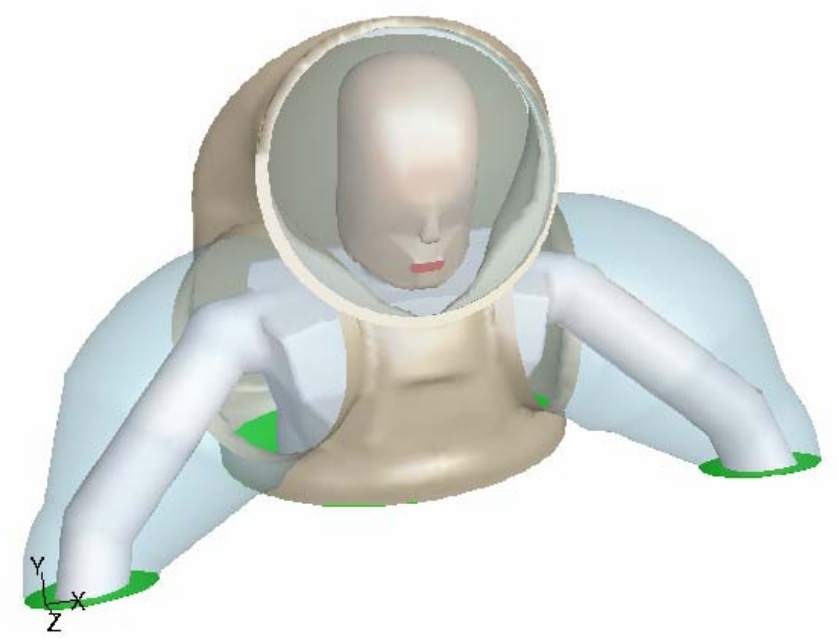

Figure 9: Complete geometry 
The first simulation cases were done to compare the model performance (without a mask) against actual hemispherical helmet test data from 1991 [7]. These tests were performed with a suited crewmember working at various metabolic rates, with the intent of measuring $\mathrm{CO}_{2}$ levels close to the mouth of the crew member. Numerous graphs were generated from these tests of $\mathrm{CO}_{2}$ levels near the mouth as functions of metabolic rates. Before simulating any of the proposed new design options for the EMU, the model was run under the same conditions as the 1991 test to see how well inhaled $\mathrm{CO}_{2}$ levels compared with the 1991 test data.

Exhaled breath was approximated using a sinusoidal relationship representing inhaling and exhaling. Concentrations of water vapor and carbon dioxide in the exhalant set to constant levels based on information from the Metabolic Man Program [4]. Breathing frequency was varied to represent changes in metabolic rates.

Incoming air entered the suit from above and behind the head. For all of the simulations described in this report, only one inlet configuration was used, consisting of four of the approximately 0.5 in $\times 1.0$ in inlet sections grouped together at the top of the HUT. The actual inlet surface area measured $2.14 \mathrm{in}^{2}$. A velocity at this inlet boundary was set to provide the necessary incoming flow rate of $3 \mathrm{cfm}$ to $6 \mathrm{cfm}$. Suit air exited the domain at the ends of the arms and around the trunk of the human using pressure outlet boundary conditions. These surfaces can be seen as the green areas of Figure 9.

\subsection{Direct Comparison of CFD Results with 1991 Test}

During the $1991 \mathrm{EMU}$ test, suit gage pressure during the test was 8.0 psig, and the test was conducted in an ambient 14.7 psia environment, so the absolute pressure in the suit model was set to 22.7 psia for these comparisons. The model used the same incoming air makeup of $22 \%$ oxygen and $78 \%$ nitrogen. Table 5 lists the results of the model along with test results for the 4 different cases run.
Table 5: Comparison with identical conditions as 1991 test

\begin{tabular}{|l|l|l|l|}
\hline $\begin{array}{l}\text { Metabolic } \\
\text { rate } \\
\text { Btu/hr }\end{array}$ & $\begin{array}{l}\text { Inflow } \\
\text { cfm }\end{array}$ & $\begin{array}{l}\text { CFD Model avg } \\
\text { inhaled } \mathrm{CO}_{2} \\
\text { concentration } \\
\% \text { at STP }\end{array}$ & $\begin{array}{l}\text { 1991 Test Min } \\
\text { Oronasal } \\
\text { CO }_{2} \\
\text { concentration } \\
\% \text { at STP) }\end{array}$ \\
\hline 600 & 3 & $0.62 \%$ & $0.50 \%$ \\
\hline 2000 & 3 & $1.49 \%$ & $1.80 \%$ \\
\hline 600 & 6 & $0.34 \%$ & $0.25 \%$ \\
\hline 2000 & 6 & $0.71 \%$ & $0.80 \%$ \\
\hline
\end{tabular}

The results showed generally a good correlation with the test results. It was noted in all the comparisons with the 1991 test results that at the lower metabolic rates of 400$600 \mathrm{Btu} / \mathrm{hr}$, the model $\mathrm{CO}_{2}$ concentrations were slightly higher. The author theorized that the reason for this was related to the sinusoidal breathing pattern. With the sinusoidal pattern, the inhale starts immediately after the exhale. There is not much time for the concentration directly in front of the mouth to decrease compared to a surface that was 1 or 2 inches in front of the mouth, as was done in the test. Right at the mouth surface would also be last location on the inhale portion of the breath to start being washed out by the $0 \% \mathrm{CO}_{2}$ fresh air stream, whereas a surface 1 or 2 inches in front of the mouth would start seeing lower $\mathrm{CO}_{2}$ levels earlier during the inhale portion of the breath.

The model results continued to show a good correlation with the test data at all metabolic rates and inflow rates. The model results supported the recommendation that came out of the 1991 test that $3 \mathrm{cfm}$ would not be sufficient to maintain the inhaled $\mathrm{CO}_{2}$ concentration below the $1.0 \%$ requirement at a metabolic rate of 1600 Btu/hr. Figure 11 shows an iso-surface of $1.0 \% \mathrm{CO}_{2}$ inside the helmet right at the beginning of the inhale portion of the breath. This was for the $1000 \mathrm{Btu} / \mathrm{hr}, 4 \mathrm{cfm}$ case listed above. Concentrations greater than $1.0 \%$ would exist within the area bounded by the iso-surface and the canopy directly in front of the crew face. 


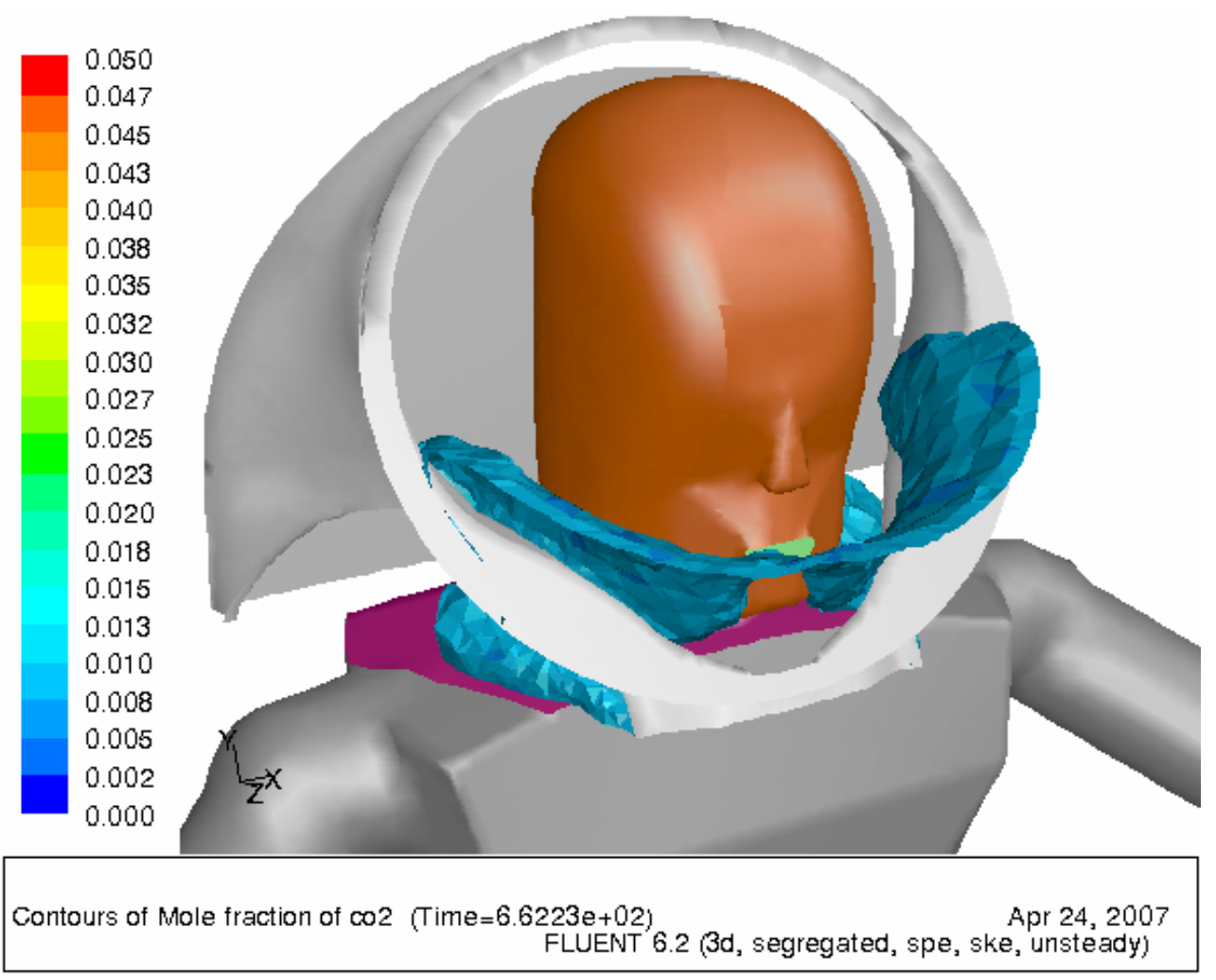

Figure 11: Iso-surface of $1.0 \%$ at STP CO2, $1000 \mathrm{Btu} / \mathrm{hr}, 4 \mathrm{cfm}$

\subsection{CFD Results for 4.3 psia Suit with Mask}

NASA was interested in determining if a mask located in front of the crew face could be used to pull out the high $\mathrm{CO}_{2}$ air in that area, instead of letting the $\mathrm{CO}_{2}$ filter down to the arms and trunk before being circulated to whatever $\mathrm{CO}_{2}$ removal hardware was used. This interest was based upon the assumption that if air with a higher concentration of $\mathrm{CO}_{2}$ was sent to the $\mathrm{CO}_{2}$ removal hardware, the mass of such hardware may be able to be reduced. The CFD model was used to investigate this by placing a surface in front of the mouth that was used to remove air from the domain directly. Because of the complexity involved with modifying any of the HUT surfaces, the simplest solution for the model was to place this mask surface on the surface of the helmet canopy and allow it to pull air directly out of the domain. The incoming air flow rate for these cases was fixed at $100 \%$ oxygen and $3 \mathrm{cfm}$, while the mask surface boundary condition (a negative velocity) was also set to pull out $3 \mathrm{cfm}$. The surfaces at the ends of the arms and trunk that previously had allowed air to leave the domain were sealed off so that the only exit location for air in the model was at the mask. A picture of the geometry showing the mask size and location is shown in Figure 12. The mask was an oval approximately 3 inches high and equal to the width of the human face.

Figure 13 shows the $1000 \mathrm{Btu} / \mathrm{hr}$ mask case at the beginning of an inhale cycle. Comparison with Figure 11 shows a much smaller area of $1.0 \% \mathrm{CO}_{2}$ and higher concentrations directly in front of the crew face.

The results for the mask cases are shown in Table 7.

Table 7: Results with mask

\begin{tabular}{|l|l|l|}
\hline $\begin{array}{l}\text { Metabolic rate } \\
\text { Btu/hr }\end{array}$ & $\begin{array}{l}\text { Inflowl } \\
\text { outflow } \\
\text { cfm }\end{array}$ & $\begin{array}{l}\text { CFD Model } \\
\text { inhaled } \\
\text { concentration } \\
\% \text { at STP }\end{array}$ \\
\hline 1000 & $3 / 3$ & $0.49 \%^{*}$ \\
\hline 1600 & $3 / 3$ & $0.77 \%^{*}$ \\
\hline
\end{tabular}

* - estimated based on rerun of results 


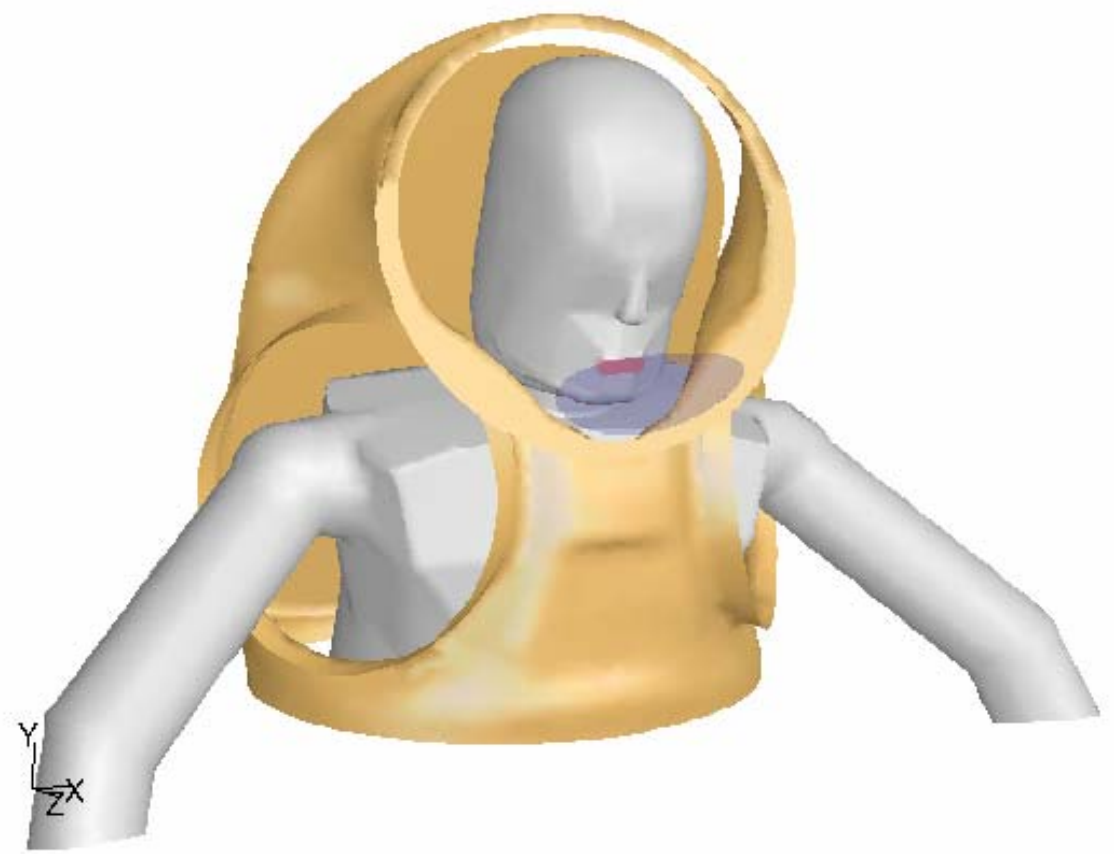

Figure 12: EMU geometry with mask

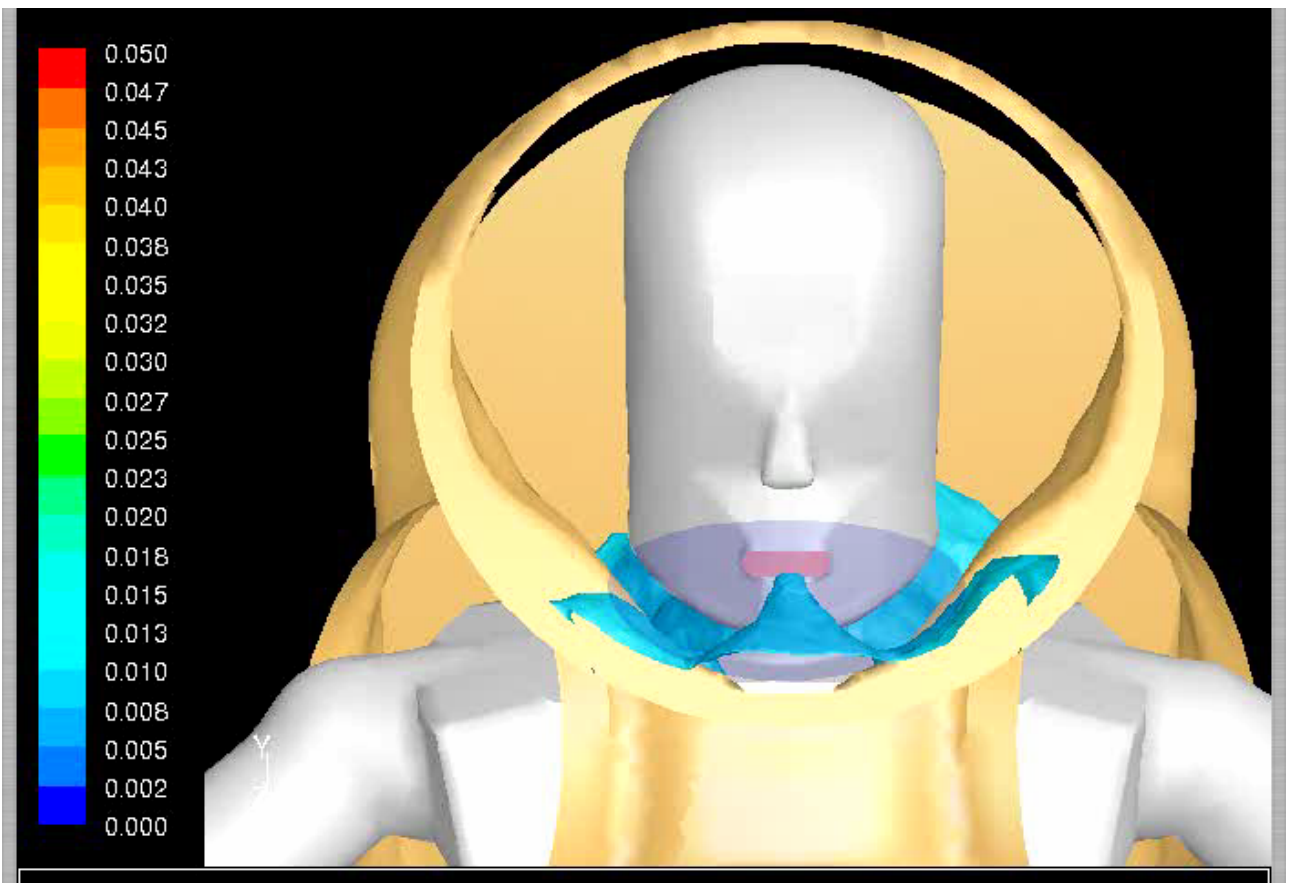

Figure 13: $1000 \mathrm{Btu} / \mathrm{hr}$, with mask at the beginning of inhalation

At the $1000 \mathrm{Btu} / \mathrm{hr}$ metabolic rate, the mask showed a reduction in the inhaled $\mathrm{CO}_{2}$ concentration over the nomask case from $0.80 \%$ down to $0.52 \%$. The $1600 \mathrm{Btu} / \mathrm{hr}$ case was run specifically to see if the mask could reduce the average inhaled $\mathrm{CO}_{2}$ concentration below the $1.0 \%$ requirement at that metabolic rate, and the results showed that the mask did accomplish this. The mask results showed that the mask with $3 \mathrm{cfm}$ performed about the same as the no-mask cases with $4 \mathrm{cfm}$ of breathing gas supply.

\subsection{CFD Model Conclusions}

CFD models with two different $\mathrm{CO}_{2}$ capture techniques were generated to simulate a breathing human inside the EMU. Comparing the average inhaled $\mathrm{CO}_{2}$ concentration, the model results showed a very good correlation with test data with human subjects at similar metabolic rates. The model also showed that a mask 
pulling air out of the EMU in front of the mouth has the potential to reduce the fresh air flowrate needed to maintain satisfactory conditions. Specifically, the model showed that a non-contact mask was capable of maintaining the average inhaled $\mathrm{CO}_{2}$ concentration below $1.0 \%$ at a $1600 \mathrm{Btu} / \mathrm{hr}$ metabolic rate with only 3 $\mathrm{cfm}$ of inlet breathing gases. Current EMU configurations without masks require a minimum of 4 $\mathrm{cfm}$ to maintain the same levels.

\subsection{RCA Sizing}

The rapid cycling amine (RCA) components for each of the evaluated configurations are sized by first using the procedures from ESCG-4470-06-TEAN-DOC-0031 [11], then rescaling the results based on updated information from Hamilton Sundstrand [12].

\subsection{Critical Comparison of PLSS Configuration Options}

The following discussion compares the advantages and disadvantages of the alternative PLSS configurations, relative to the modified baseline, considered in this study.

\subsection{Open Loop Full Contact Mask}

The primary advantage of the open loop full contact mask configuration is the simplification of the design incurred by removing the need for $\mathrm{CO}_{2}, \mathrm{H}_{2} \mathrm{O}$, and trace gas removal equipment by exhausting all exhaled gases to space. The increased mass and volume required to store the extra $\mathrm{O}_{2}$ that is vented into space overtakes the mass and volume reductions associated with the omission of the $\mathrm{CO}_{2}, \mathrm{H}_{2} \mathrm{O}$ and trace gas treatment equipment, producing a $10.4 \mathrm{~kg}$ increase in mass and a $0.030 \mathrm{~m}^{3}$ increase in volume over the modified baseline.

In the event of a mask failure, the backup oxygen supply system for this configuration is an open loop suit/helmet purge. This requires a scheme for mechanically separating the full contact mask form the astronaut's face without the use of his/her hands.

\subsection{Closed Loop Full Contact Mask}

The closed loop full contact mask configuration is the most complex of those compared in this study. This complexity is primarily caused by the requirement of temporary breathing gas storage, implemented using two expandable counterlungs, due to the non-steady nature of human breathing. One of the counterlungs is used to store exhaled gases, while the other stores treated inhalation gases.
A complication arising from use of counterlungs in EVA applications involves their location with respect to the pressurized suit. If the counterlungs are mounted within the pressurized suit environment, the periodic expansion and contraction of the counterlungs will tend to cause unwanted variations in the suit pressure. This effect will be lessened by the opposing expansion and contraction of the astronaut's chest and the flexing of the suit material. If the counterlungs are located outside the pressurized suit interior, the pressure differential between the gases inside the counterlungs and the vacuum of space will tend to force the counterlungs into the fully inflated position at all times. In order to counteract the effect of the pressure differential, the counterlungs would first need a semi-rigid framework to restrict to expansion to a single, controllable direction. This requirement could be satisfied using bellows. Next, a position-independent counterforce, such as that provided by a constant-force spring, would be required to balance the pressure differential between the counterlung interior and exterior. Note, however, that if the counterforce is provided by a constant-force spring or any other non-adjustable device, then the counterlung will only function properly at the designed pressure differential.

In addition to these common observations, several factors associated with specific counterlung configurations are noted:

\subsubsection{Decoupled Constant Fan Speed Counterlungs}

The advantage of using decoupled constant fan speed counterlungs is that the pressure drop associated with the $\mathrm{CO}_{2}, \mathrm{H}_{2} \mathrm{O}$, and trace gas removal components is overcome by a constant speed fan, reducing the effort of breathing. However, because the fan runs at a constant speed, off-design variations in the astronaut's average ventilation rate, which are caused by metabolic rate changes, will produce variations in the breathing resistance. Additionally, the effort of breathing will increase in the event of a fan failure, possibly accompanied by unstable counterlung operation.

\subsubsection{Coupled Fanless Counterlungs}

The coupled fanless counterlung configuration is intended to be purely lung-powered, with the inhalation and exhalation counterlungs mechanically constrained to expand and contract together. The ventilation gas speed through the $\mathrm{CO}_{2}, \mathrm{H}_{2} \mathrm{O}$, and trace gas treatment components would vary according to a periodic pattern. Relative to the fan-based counterlung configurations, mass and volume savings are obtained due to the omission of the fan and, secondarily, through reduced power supply and thermal control system sizes resulting from the decreased power usage. Because no fan is 
used, the effort of breathing would be more difficult than that of the fan-powered configurations.

\subsubsection{Breathing Bags}

The breathing bag configuration is similar to the decoupled constant fan speed counterlung configuration in that a constant speed fan is used to drive the breathing gases from the inhalation bag, through the $\mathrm{CO}_{2}, \mathrm{H}_{2} \mathrm{O}$, and trace gas removal components, to the exhalation bag. The primary difference is that, due to their unconstrained flexibility, the breathing bags can only be located within the pressurized suit environment. The primary advantage of the breathing bag configuration over the other counterlung configurations is that large flat breathing bags can be used as a liner inside the space suit, thus adding no extra volume to the on-back PLSS. However, since the flexible bags will be subject to the unpredictable motion of the astronaut's body, the likelihood of unforeseen and unrepairable damage during an EVA is apparently higher. Additionally, the functionality of the breathing bags could be impaired if pressure is applied to the side of the suit containing the bag: if, for example, an astronaut on lunar EVA falls onto the section of his suit that houses a breathing bag. Like the decoupled constant fan speed counterlung configuration, a constant speed fan reduces the required breathing force and does not adjust for metabolic variations in breathing rate without changing the resistance to breathing. Also, the event of a fan failure would increase the breathing difficulty and possibly impair the design functionality of the breathing bag system.

Though all counterlung configurations described above were considered for this study, quantitative sizing was performed only on the decoupled constant fan speed counterlungs. The other configurations were not sized because the extra breathing effort associated with the coupled fanless counterlungs is not desirable for EVAs that can last up to 8 hours and because the potential functionality impairment of breathing bags due to unforeseen contact pressure and/or snagging, combined with their lack of maintenance access, casts doubt on their reliability for critical EVA applications.

The decoupled constant fan speed counterlung variant of the closed loop full contact mask configuration weighs only $0.11 \mathrm{~kg}$ less than the modified baseline configuration but requires $0.0077 \mathrm{~m}^{3}$ more volume.

\subsection{Closed Loop Non-Contact Mask}

The closed loop non-contact mask configuration is nearly identical to the modified baseline configuration, except that the exhaled gases are extracted from directly in front of the astronaut's mouth instead of at the body's extremities. This method of extraction reduces the mixing of exhaled $\mathrm{CO}_{2}$ with inhalation gases and increases the $\mathrm{CO}_{2}$ concentration in the RCA inlet stream. For a hemispherical helmet, the mixing reduction lowers the $\mathrm{O}_{2}$ flowrate needed for $\mathrm{CO}_{2}$ washout from $113 \mathrm{alpm}$ ( $4 \mathrm{acfm}$ ) to $85 \mathrm{alpm}$ ( $3 \mathrm{acfm}$ ). The ventilation fan size is thus downsized as a result of this flowrate reduction. The lower power consumption of the smaller ventilation fan leads to battery size and thermal control system mass and volume reductions. Also, the increased $\mathrm{CO}_{2}$ concentration in the RCA inlet gases leads to a reduction in required RCA size and mass. The net results are that the closed loop noncontact mask configuration requires $0.62 \mathrm{~kg}$ less mass and $0.0019 \mathrm{~m}^{3}$ less volume than the modified baseline, making this the preferred alternative in terms of mass and volume requirements. The disadvantage of this configuration is the added complexity of mounting the non-contact mask in the helmet and routing the exhalation gas lines from the helmet interior to the backpack PLSS.

\subsection{Additional Observations}

In comparing the mask-based PLSS concepts with the modified baseline configuration, several issues beyond mass and volume are considered. Many of the following issues were identified by the Constellation Space Suit Element (CSSE) team [15].

\subsubsection{Performance}

In evaluating mask-based PLSS configurations, items affecting the equipment functionality and performance must be considered.

\subsubsection{Convective/Evaporative Cooling}

The mask-based configurations eliminate the flow of gases over the astronaut's body during normal operations, thus eliminating the associated convective cooling. Under these conditions, liquid cooling serves as the only method of removing excess heat from the suit environment. Because current suit system and LCVG designs do not apply liquid cooling to hands and feet, the lack of convective cooling may cause discomfort in these extremities.

Each mask-based configuration includes a suit-mounted purge valve, which permits oxygen to flow through the LCVG. This provides up to approximately $185 \mathrm{~W}$ of convective/evaporative cooling for the astronaut's body in the event of a liquid cooling system failure, but should only be used during the emergency termination of an EVA due to the rapid depletion of $\mathrm{O}_{2}$ due to purging. The $185 \mathrm{~W}$ estimate is based on a hot case where dry 
oxygen enters the EMU at $305 \mathrm{~K}$ and leaves the EMU fully saturated.

\subsubsection{Secondary Oxygen Package (SOP) Sizing and Duration}

All configurations compared in this study incorporate a secondary oxygen package that is sized to provide approximately 30 minutes of oxygen in purge mode at $113 \mathrm{alpm}$ (4 acfm). If the primary oxygen supply for a full-contact mask configuration fails, using secondary oxygen through the mask will extend the SOP duration to $2.5 \mathrm{~h}$ at a metabolic rate of $300 \mathrm{~W}(1000 \mathrm{Btu} / \mathrm{h})$ or $1.26 \mathrm{~h}$ at a metabolic rate of $600 \mathrm{~W}(2000 \mathrm{Btu} / \mathrm{h})$. For the non-contact mask configuration, the SOP supply could last for 43 minutes, due to the reduction in $\mathrm{CO}_{2}$ wash-out flowrate from $113 \mathrm{alpm}$ (4 acfm) to $85 \mathrm{alpm}$ (3 acfm). These extended SOP lifetimes can only be realized if the liquid cooling system is either properly functioning or is unneeded due to cold-to-moderate thermal surroundings and if the suit has no significant punctures or leakages. Because the SOP must be sized to accommodate leakages and thermal control system failures, no associated mass or volume savings are realized by the mask configurations.

\subsubsection{Nitrogen Purge}

The possibility of reducing the pre-EVA $\mathrm{N}_{2}$ purge duration for the full-contact mask configurations has been explored. This reduction would be obtained by purging only the breathing loop, not the suit interior. However, various emergency scenarios would require that the mask be doffed and the suit interior be used as a breathing gas environment. The presence of unpurged $\mathrm{N}_{2}$ in the suit interior in this eventuality could reduce the oxygen concentration to unsafe levels and increase the risk of developing the bends. Displacement of oxygen from the helmet would be particularly pronounced within gravity environments due to buoyancy effects caused by $\mathrm{N}_{2}$ having a lower molecular mass than $\mathrm{O}_{2}$ and by thermally-induced density differences caused by the introduction of colder oxygen into a suit containing nitrogen that had been warmed by the astronaut's body heat. Because of these complications, the entire suit interior must be purged in both the mask-based configurations and the non-mask configurations. No pre-EVA $\mathrm{N}_{2}$ purge duration savings is obtainable.

\subsubsection{Communications}

The use of a mask would require that a microphone be mounted inside the mask to enable communications. This would require additional cables and electrical coupling beyond those required for the current "snoopy" cap implementation. Additionally, based on communications quality noted in flight masks used in the JSC B7 Dual Glove Box (DGB) tests, pressure compensation and digital filtering would likely be required to improve the communications quality to the level achieved by with the current EMU.

\subsubsection{Human Factors}

The use of mask-based PLSS configurations introduces several issues involving the interaction between the astronaut and the suit system.

\subsubsection{Mask Sizing}

Use of a full-contact mask may require a custom mask for each EVA crewmember to reduce the leakage of oxygen through the seal around the face. Also, additional preparation time would be required prior to each EVA to properly install and configure the mask.

\subsubsection{Restriction of Movement}

A mask installed inside the helmet of a space suit would likely add restrictions to head movements that would otherwise aid peripheral sight. The movement restrictions would result from the presence of feed lines that supply gases to and from the mask. These feed lines are affixed to the same anthropomorphic vessel that constrains the astronaut's body.

\subsubsection{Drinking Water}

Presently, $0.95 \mathrm{~L}$ (32 fl oz) of drinking water is supplied by the EMU using a feedport from a Disposable In-suit Drink Bag (DIDB). Coverage of the astronaut's face with a full-contact mask would block access to any such feedports and increase the complexity of implementing a system for supplying drinking water to the astronaut during EVA.

\subsubsection{Valsalva}

Implementation of a hands-free valsalva operation in conjunction with an EMU mask would be difficult. The method used in the current EMU, involving a piece of foam rigidly mounted to the neck ring, would not be possible if a mask were covering the oral/nasal region. For the masks and helmets used in the JSC B7 Dual Glove Box (DGB) facility, valsalva is accomplished by pinching the mask. This would also not be feasible in an EMU. 


\subsubsection{Safety}

Various safety-related issues must be addressed if a mask-based EMU design is implemented.

\subsubsection{Mask Fit}

Experience in the JSC B7 Dual-Glove Box (DGB) facility, in which flight helmets and masks are used for prebreathe and up to 6 hour "EVA" sessions in the $11 \mathrm{ft}$ outer-lock, has shown that fit during the session is often uncomfortable for the wearer and required adjustment during the run. Such adjustments would not be possible in an EMU, resulting in possible injury to the face/nose or uncomfortable drying of the eyes due to blow-by.

\subsubsection{Inadvertent Disconnection}

Because full-contact mask configurations would likely require custom masks, fitted for each crewmember, quick-disconnects (QD) will be required to install and remove the mask from the EMU. During the EVA, relative motion between the crewmember and the EMU poses a risk of inadvertent disconnection of a QD. Without an emergency procedure in place, such an event would be life-threatening. For the mask-based configurations considered in this study, an inadvertent disconnect of a mask feed-line QD would be handled by removing the mask from the crewmember's face, placing the suit on $\mathrm{O}_{2}$ purge mode, and aborting the EVA.

\subsubsection{Vomitus}

The event of an EVA crewmember vomiting produces the risk of clogging the breathing passages. If all vent ports are clogged, then the crewmember's breathing process will be impaired. The more vent ports are available, the less likely that all vent ports will be clogged. The modified baseline contains four vent ports in the LCVG and a helmet purge valve for certain emergency conditions. The mask-based configurations each have a single vent port for normal operations. In the event that this vent port becomes clogged with vomitus, an emergency mode would be activated, in which either the LCVG vents or the helmet purge valve would be used to maintain non-mask breathing gas circulation while aborting the EVA.

\subsubsection{Loss of Communications}

In the event of a communications failure, hand signals are used to send messages. Additionally, crewmembers observe each other's facial expressions to relay information, including health status. The presence of a face mask would restrict the view of the facial features, limiting the feedback to fellow crewmembers and observers.

\subsection{Conclusions and Recommendations}

In assessing the relative merits of the modified baseline, open loop full contact mask, closed loop full contact mask, and closed loop non-contact mask PLSS configurations, associated mass and volume requirements were the primary criteria in the present study. Other criteria related to design complexity, performance, human factors, and safety considerations were briefly considered, but were not thoroughly explored.

None of the full-contact mask configurations studied offered a significant mass or volume advantage over the modified baseline configuration. The open loop full contact mask system mass and volume are significantly larger than the modified baseline equivalents because of its inefficient handling of $\mathrm{O}_{2}$. The mass of the closed loop full contact mask configuration, assuming independent constant fan speed counterlungs, is approximately $0.11 \mathrm{~kg}$ less than the modified baseline, but this is accompanied by a $0.0077 \mathrm{~m}^{3}$ increase in volume. The small mass reduction does not justify the added volume requirement and the added complexity that would be required to implement the closed loop full contact mask configuration. Additionally, most of the technical difficulties described in section 4.4 of this report apply primarily to the use of full-contact masks.

From a mass and volume perspective, the most favorable of the mask-based configurations is the closed loop non-contact mask. The associated mass and volume savings over the modified baseline are $0.62 \mathrm{~kg}$ and $0.0019 \mathrm{~m}^{3}$, respectively. Also, because the mask does not make contact with the astronaut's face, many of the pitfalls of using a full-contact mask in a closed space suit environment are avoided, such as the need to retract the mask for emergency breathing operations, the need for using custom-fitted masks, and the blocked access to the oral/nasal area that restricts access to drinking water feedports and valsava-enabling devices. The presence of the non-contact mask in front of the astronaut's face would still likely limit head movement, visibility, and a view of the astronaut's face that would provide feedback in the event of a communications failure. Noise levels within the helmet may also be increased due to the channeling of the entire $113 \mathrm{alpm}$ (4 acfm) flow through an open mask located directly in front of the astronaut's face. Also, the routing of supply and return lines between the helmet-mounted mask and the back-mounted PLSS would add complexity to the system.

Due to these considerations, the modified baseline configuration is recommended over the three maskbased configurations considered. The closed loop non- 
contact mask configuration offers minimal advantages and adds complexity. The full-contact mask configurations do not offer any significant mass or volume savings and introduce many technical disadvantages that could impair the performance, comfort, and safety of the EMU.

\section{References}

1. Bailey, Paul, "Constellation Suit Element Portable Life Support System (PLSS) Schematic Selection Study," NASA JSC-65443, CTSD-CX0005, February 2007.

2. Gilbert, John and Susan Schentrup, "Determination of Residual Carbon Dioxide During Forced Ventilation of a Hemispherical Space Suit Helmet - Final Test Report," NASA/JSC, January 31, 1991.

3. "Air Bearing Fan - Final Test Report," Document No. CTSD-SS-608, Crew and Thermal Systems Division, NASA-Lyndon B. Johnson Space Center, Houston, TX: December 31, 1992.

4. Bue, Grant, Computer Program Documentation 41-Node Transient Metabolic Man Program, Lockheed Engineering and Sciences Company, Inc., LESC-27578, CTSD-0425, October, 1989.

5. Webb, P., Bioastronautics Data Book, NASA SP-3006, Web Associates, NASA, 1964.

6. Personal communications with Cory Smith of Halcyon, 2007.

7. J. Gilbert, S. Schentrup, "Determination of Residual Carbon Dioxide During Forced Ventilation of a Hemispherical Space Suit Helmet", Final Test Report, 31 January, 1991.

8. Space Shuttle Extravehicular Mobility Unit Space Suit Assembly (SSA) Mini-Databook, Table 9: LCVG Specifications, Page 127.

9. Fox, Robert W. and Alan T. McDonald, Introduction to Fluid Mechanics, 3rd ed., John Wiley and Sons: New York, 1985.
10. Avallone, Eugene A. and Theodore Baumeister III, Mark's Standard Handbook for Mechanical Engineers, 9th ed., p. 14-54, McGraw Hill: New York. 1986.

11. Conger, B. C., et. al., "Life Support Commonality across the Lunar Sortie Architecture," Jacobs ESCG/NASA, ESCG-4470-06-TEAN-DOC0031, May 2006.

12. Papale, William and Heather L. Paul, "Development status of an EVA-sized cycling amine bed system for spacesuit carbon dioxide and humidity removal," International Conference on Environmental Systems, SAE Paper No. 2007-01-3272, July 2007.

13. Man-Systems Integration Standards, NASASTD-3000, Revision B, July 1995, Section 14.2.3.9.

14. Brown C. B. and B. M. Miranda, "Extravehicular Activity System Sizing Analysis Tool (EVAS_SAT) Version 3.0 User's Guide," ESCG4470-06-TEAN-DOC-0105, August 31, 2006.

15. Campbell, Colin, E-mail to Rose Bitterly and Heather Paul on Thursday, August 30, 2007 at 1:23 PM (CST). 


\section{Acronyms and Abbreviations}

\begin{tabular}{|c|c|}
\hline ABF & Air Bearing Fan \\
\hline acfm & Actual Cubic Feet per Minute \\
\hline alpm & Actual Liters per Minute \\
\hline CFD & Computational Fluid Dynamics \\
\hline CSSE & Constellation Space Suit Element \\
\hline DGB & Dual Glove Box \\
\hline DIDB & Disposable In-suit Drink Bag \\
\hline EMU & Extravehicular Mobility Unit \\
\hline ESCG & $\begin{array}{l}\text { Engineering and Science } \\
\text { Contract Group }\end{array}$ \\
\hline EVA & Extravehicular Activities \\
\hline EVAS_SAT & $\begin{array}{l}\text { Extravehicular Activity System } \\
\text { Sizing Analysis Tool }\end{array}$ \\
\hline HECS & $\begin{array}{l}\text { Helmet Exhalation Capture } \\
\text { System }\end{array}$ \\
\hline HUT & Hard Upper Torso \\
\hline JSC & Johnson Space Center \\
\hline LCVG & $\begin{array}{l}\text { Liquid Cooling and Ventilation } \\
\text { Garment }\end{array}$ \\
\hline NASA & $\begin{array}{l}\text { National Space and Aeronautics } \\
\text { Administration }\end{array}$ \\
\hline OSS & Oceaneering Space Systems \\
\hline PLSS & Portable Life Support System \\
\hline QD & Quick-Disconnect \\
\hline RCA & Rapid Cycling Amine \\
\hline SOP & Secondary Oxygen Package \\
\hline STEP & $\begin{array}{l}\text { STandard for the Exchange of } \\
\text { Product model data }\end{array}$ \\
\hline STP & $\begin{array}{lll}\text { Standard } & \text { Temperature } & \text { and } \\
\text { Pressure } & & \end{array}$ \\
\hline SWME & Suit Water Membrane Evaporator \\
\hline TEES & $\begin{array}{l}\text { Texas Engineering Experiment } \\
\text { Station }\end{array}$ \\
\hline
\end{tabular}

